\title{
The Brazilian species of the family Aristeidae Wood-Mason (Crustacea: Decapoda)
}

\author{
F. D'INCAO
}

Departamento de Oceanografia-FURG, Caixa Postal 474, 96201.900 Rio Grande, RS, Brazil

(Accepted 8 May 1998)

The Brazilian Aristeidae are reviewed and shown to be represented by the following species: Aristaeomorpha foliacea (Risso, 1827); Hepomadus tener Smith, 1884; Plesiopenaeus edwardsianus (Johnson, 1867); Benthesicymus bartletti Smith, 1882; Gennadas brevirostris Bouvier, 1905; and Bentheogennema intermedia (Bate, 1888). Of these, A. foliacea, $H$. tener, B. bartletti and G. brevirostris are new records for Brazil. All of the species are widely distributed in the Atlantic and Indo-West Pacific oceans. Gennadas brevirostris is restricted to the Atlantic and Hepomadus tener occurs in the Western Atlantic and Indo-West Pacific oceans. Bentheogennema intermedia is the only species recorded for the Eastern Pacific. The latitudinal distribution among them ranges between $50^{\circ} \mathrm{N}$ and $40^{\circ} \mathrm{S}$.

Keywords: Aristeidac, distribution, Brazil.

\section{Introduction}

The family Aristeidac is poorly represented in Brazil and the following institutions do not have any specimens in their crustacean reference collections: Universidade Federal da Paraiba; Departamento de Oceanografia, Universidade Federal de Pernambuco; Museu Nacional, Universidade Federal do Rio de Janeiro; Museu de Zoologia and Universidade de São Paulo. This is duc to the lack of deep water (over $600 \mathrm{~m}$ ) research in Brazil.

Only two Aristeidae species are cited from Brazil in the literature. Plesiopenaeus edwardsianus (Johnson, 1867) from Amapá (Bullis and Thompson, 1959) and Bentheogennema intermedia (Bate, 1888) from Pernambuco (Bate, 1888; Burkenroad, 1936; Coelho and Ramos, 1972; Ramos-Porto et al., 1989). Plesiopenaeus edwardsianus is recorded from Uruguay at $35^{\circ} 44^{\prime} \mathrm{S} 52^{\circ} 05^{\prime} \mathrm{W}$ (Itusarry, 1984). Three species from Argentina, collected by the Challenger Expedition (1873-1876), are noted by Bate (1888), Crosnier and Forest (1973), Pérez Farfante (1987): Plesiopenaeus armatus (Bate, 1881) and Benthesicymus brasiliensis Bate, 1881 at $35^{\circ} 39^{\prime} \mathrm{S} 50^{\circ} 47^{\prime} \mathrm{W}$ and Pseudaristeus speciosus (Bate, 1881 ) at $36^{\circ} 44^{\prime} \mathrm{S} 46^{\circ} 16^{\prime} \mathrm{W}$.

\section{Materials and methods}

The Aristeidae of the following collections were examined during this study: Laboratório de Crustáceos Decápodes, Fundação Universidade do Rio Grande (FURG); Instituto Nacional de Pesca, Uruguay (INAPE); Instituto Nacional de 
Investigación y Desarrollo Pesquero, Argentina (INIDEP) and The Natural History Museum, London (NHM).

The length of the carapace is measured in millimetres (orbital angle to posterodorsal margin of carapace) and the number and sex of individuals are indicated. The names of the countries are written in English; otherwise, all geographic features, localities and provinces are in the original idiom. The synonym lists only the main papers relating to each species. Abbreviations used: male $=\hat{s}$; female $=q$; station $=$ st.

\section{Results}

\section{Aristaeomorpha foliacea (Risso, 1827)}

(Figures 1A,2)

Penaeus foliaceus Risso, 1827: 69.

Aristeus rostridentatus Bate, 1881: 189; 1888: 317.

Aristaeomorpha foliacea: Burkenroad, 1936: 85; Crosnier, 1978: 23; 1985: 842; 1989: 42.

Aristeomorpha foliacea: Crosnier and Forest, 1973: 287; Crosnier, 1976: 227.

Material examined. Colombia: La Guajira. Off Cabo de la Vela, Oregon, st. $5688,490 \mathrm{~m}, 1$ ㅇ, $59.0 \mathrm{~mm}, 1$ ơ, $35.8 \mathrm{~mm}$, (NHM 1977.532). Brazil: Paraná, (2524'S $44^{\circ} 54^{\prime} \mathrm{W}$ ), W.Herwig, st. 83, $500 \mathrm{~m}, 1$, $29.0 \mathrm{~mm}$, (FURG-1607). Santa Catarina, ( $\left.28^{\circ} 14^{\prime} \mathrm{S} 47^{\circ} 46^{\prime} \mathrm{W}\right)$, W. Herwig, st. $137,1200 \mathrm{~m}, 1$ \%, $55.5 \mathrm{~mm}$, (INIDEP). Rio Grande do Sul, ( $\left.31^{\circ} 11^{\prime} \mathrm{S} 49^{\circ} 35^{\prime} \mathrm{W}\right)$, Atlántico Sul, st. 37, $510 \mathrm{~m}, 11 \stackrel{\circ}{\AA}, 26.7-34.6 \mathrm{~mm}$, 28 , $21.0-45.7 \mathrm{~mm}$, (FURG-0742).

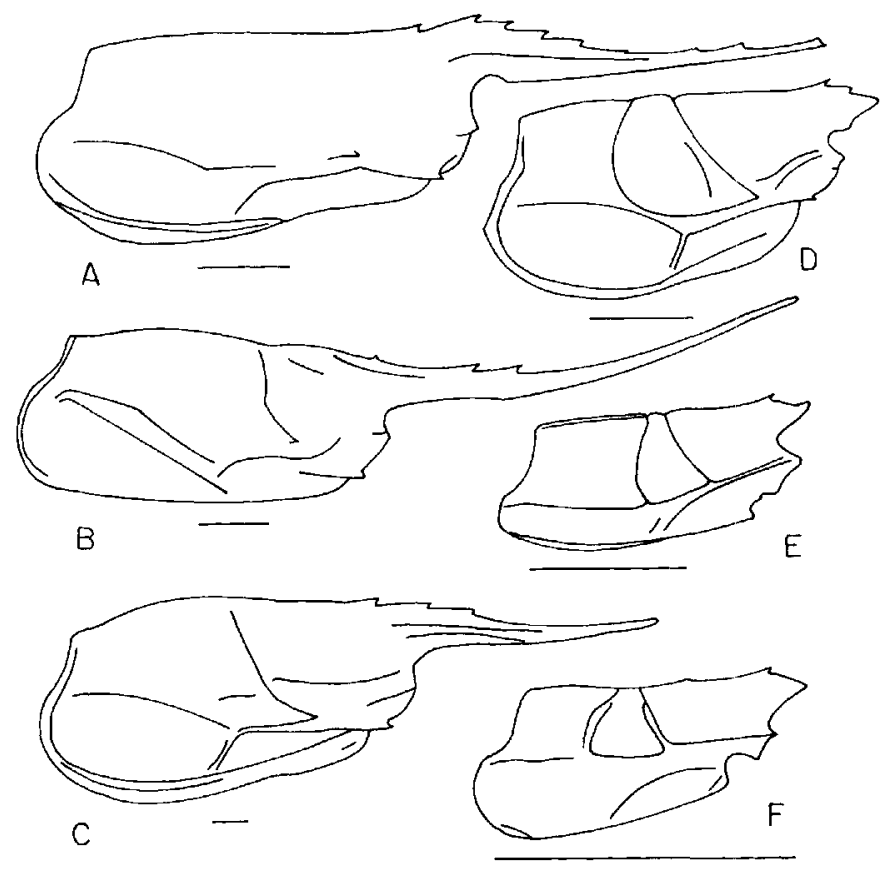

FIG. 1. Carapace of Brazilian Aristeidae shrimps: (A) Aristaeomorpha foliacea, FURG-0742; (B) Ilepomadus tener, FURG-1602; (C) Plesiopenaeus edwardsianus, FUR G-1601; (D) Benthesicymus bartletti, FURG-1605; (E) Bentheogennema intermedia, NHM-1888.22; (F) Gennadas brevirostris, FURG-1745. Scale: $1 \mathrm{~cm}$. 


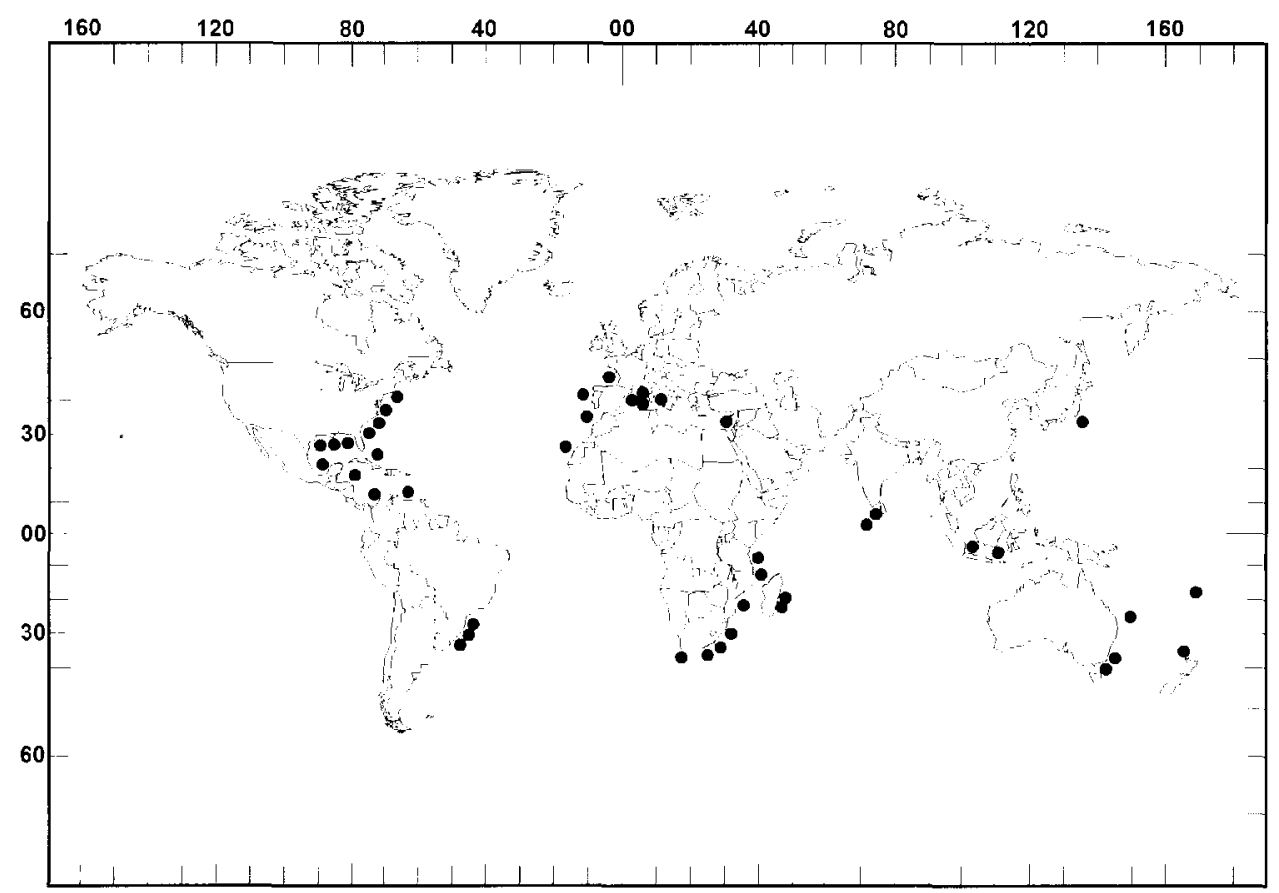

FIG. 2. Geographical distribution of Aristaeomorpha foliacea (Risso, 1827).

Type-locality. Mediterranean Sea, off Nice, France.

Distribution. Western Atlantic (northern limit: south Massachusetts, USA; southern limit: Rio Grande do Sul, $31^{\circ} 11^{\prime} \mathrm{S} 49^{\circ} 35^{\prime} \mathrm{W}$, Brazil): United States (Massachusetts, Philadelphia, Virginia, North and South Carolina, Georgia, Florida, Mississippi, Louisiana, Texas), Mexico, Bahama, Caribbean Sea, Colombia, Venezuela, Brazil (Paraná, Santa Catarina, Rio Grande do Sul). Eastern Atlantic (northern limit: Bay of Biscay, France; southern limit: Rio de Oro, Sahara): France (Bay of Biscay), Spain, Portugal, Morocco, Sahara (Rio de Oro), Mediterranean Sea (Spain, France, Italy, north coast of Africa, Algeria, Israel). Indo-West Pacific (northern limit: Yokohama, $35^{\circ} \mathrm{N}$, Japan; southern limit: Victoria, $37^{\circ} 45^{\prime} \mathrm{S}$, Australia): South Africa (Kosy Bay, Richards Bay, Durban, East London, Port Elizabeth), Mozambique (Monte Belo, Cabo Correntes), Madagascar, Tanzania (Zanzibar), Maldive Is., Sri Lanka, Indonesia (Ceram Sea, Sumatra), Japan (Yokohama, Owase, Kumononada, Sidouka), Australia (New South Wales, Victoria), New Caledonia, New Zealand (North Auckland), Fiji Is.

Ecological notes. The occurrence of this species along the Brazilian coast (Paraná, Santa Catarina and Rio Grande do Sul) has not been previously reported. The species occurs at depths between 60 and $1300 \mathrm{~m}$, on sand and mud bottoms.

\section{Hepomadus tener Smith, 1884.}

(Figures 1B,3)

Hepomadus tener Smith, 1884: 409; Burkenroad, 1936: 86; Crosnier, 1978: 47; 1985: 860.

Material examined. Venezuela: Venezuela Basin, $\left(13^{\circ} 48^{\prime} \mathrm{N} 67^{\circ} 37^{\prime} \mathrm{W}\right), 5056 \mathrm{~m}$, damaged specimen, $29.0 \mathrm{~mm}$, (NHM 1984.43); (13 $\left.51^{\prime} \mathrm{N} 67^{\circ} 52^{\prime} \mathrm{W}\right), 5046 \mathrm{~m}, 1^{\circ}$, 


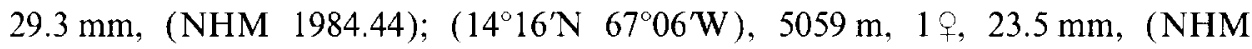
1984.45). Brazil: Rio de Janeiro. ( $\left.22^{\circ} 41^{\prime} \mathrm{S} 40^{\circ} 08^{\prime} \mathrm{W}\right)$, W. Herwig, st. $103,1200 \mathrm{~m}$, 1 ㅇ, $44.0 \mathrm{~mm}$, (INIDEP-1264); (22 $41^{\prime} \mathrm{S} 40^{\circ} 08^{\prime} \mathrm{W}$ ), W. Herwig, st. 103, $1200 \mathrm{~m}, 1$,

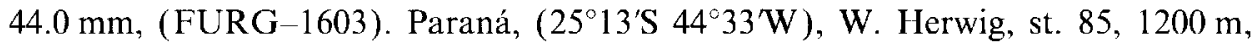
1 of, $36.5 \mathrm{~mm}, 1$, $45.7 \mathrm{~mm}$, (FURG-1602). Santa Catarina, $\left(27^{\circ} 45^{\prime} \mathrm{S} 46^{\circ} 33^{\prime} \mathrm{W}\right.$ ), W. Herwig, st. $120,1200 \mathrm{~m}, 1$ औ $, 42.5 \mathrm{~mm}, 1 \% 48.2 \mathrm{~mm}$, (INIDEP-695).

Type-locality. Off Chesapcake Bay, $37^{\circ} 12^{\prime} \mathrm{N} 69^{\circ} 30^{\prime} \mathrm{W}$, USA.

Distribution. Western Atlantic (northern limit: off Atlantic City, New Jersey, USA; southern limit: Santa Catarina, $27^{\circ} 45^{\prime} \mathrm{S} 46^{\circ} 33^{\prime} \mathrm{W}$, Brazil): United States (New Jersey, Virginia, North Carolina, Florida, Alabama, Louisiana, Texas), Mexico (Tamaulipas, Veracruz, Yucatan), Bahama (Turks Is.), Haiti (Gulf of Gonave), Tobago (Charlotteville), west Caribbean (Cayos Hobbies), Panama (off Punta Manzanillo), Colombia (off San Bernardo, Puerto Colombia, La Guajira), Venezuela, Brazil (Rio de Janeiro, Paraná, Santa Catarina). Indo-West Pacific (northern limit: Bay of Bengal; southern limit: Madagascar): Bay of Bengal, Chagos Is., Reunion Is., Madagascar.

Ecological notes. The species is a new record for Brazil and occurs at depths between 933 and $5413 \mathrm{~m}$.

\section{Plesiopenaeus edwardsianus (Johnson, 1867)}

(Figures 1C,4)

Penaeus Edwardsianus Johnson, 1867: 897.

Aristeus coralinus Bate, 1888: 32.

Plesiopenaeus edwardsianus: Burkenroad, 1936: 94; Crosnier and Forest, 1969: 550; Crosnier, 1978: 88.

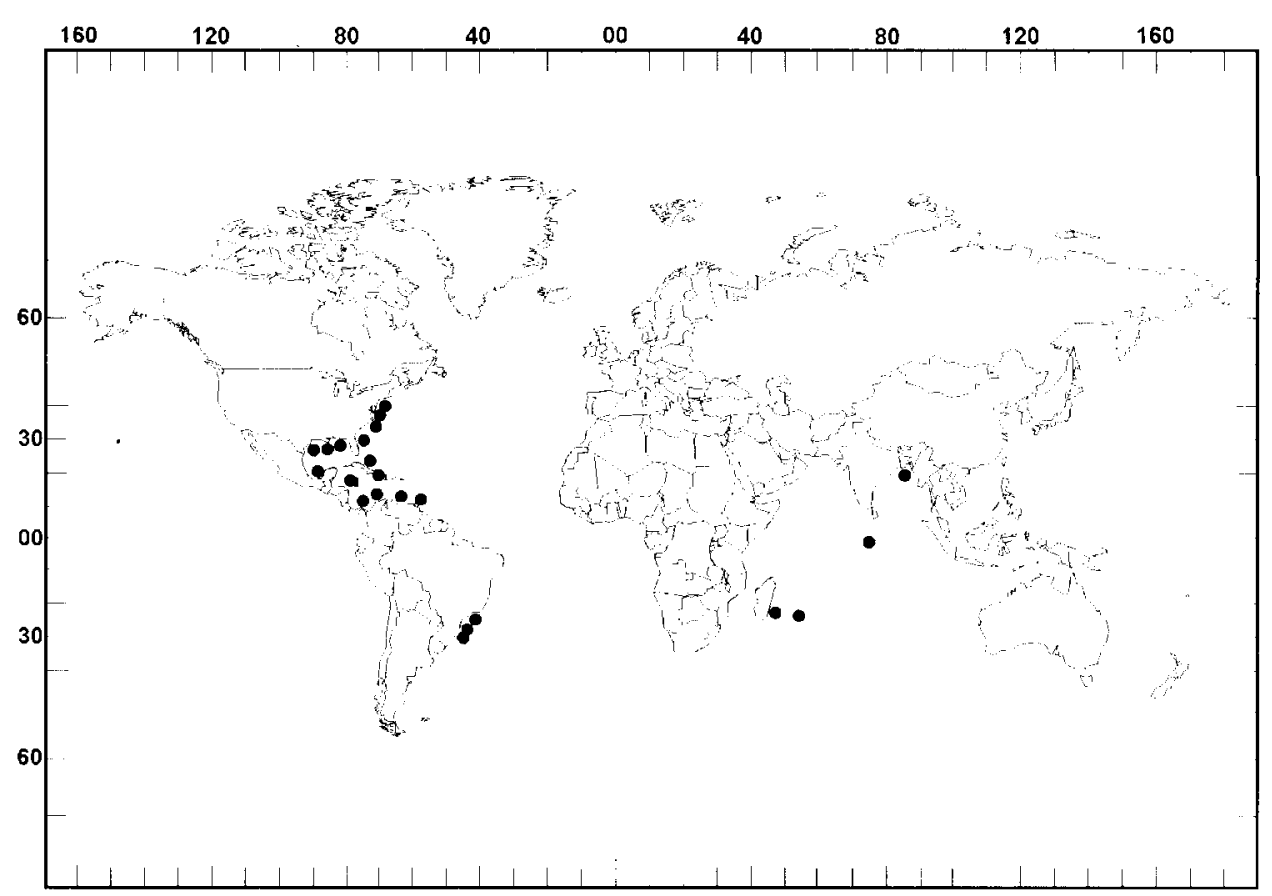

FIG. 3. Geographical distribution of Hepomadus tener Smith, 1884. 
Material examined. United States: Alabama. Off Mobile Bay, $\left(29^{\circ} 10^{\prime} \mathrm{N}\right.$ $87^{\circ} 57^{\prime} \mathrm{W}$ ), 650-730 m, $1 \hat{\circ}, 40.6 \mathrm{~mm}, 3$ ㅇ, 64.6-84.1 mm, (NHM 1977.533). Brazil: Santa Catarina $\left(26^{\circ} 43^{\prime} \mathrm{S} 46^{\circ} 07^{\prime} \mathrm{W}\right)$, W. Herwig, st. $117,800 \mathrm{~m}, 1$ \%, $74.3 \mathrm{~mm}$, (INIDEP); $\left(26^{\circ} 43^{\prime} \mathrm{S} 46^{\circ} 07^{\prime} \mathrm{W}\right)$, W. Herwig, st. $117,800 \mathrm{~m}, 2$ \% $68.6-75.2 \mathrm{~mm}$, (FURG-1601). Uruguay. (35'14'S 52 06'W), B/P Lerez, st. $820511,700 \mathrm{~m}$, (INAPE).

Type-locality. Madeira.

Distribution. Western Atlantic (northern limit: off Newfoundland, $43^{\circ} 30^{\prime} \mathrm{N}$, Canada; southern limit: $35^{\circ} 34^{\prime} \mathrm{S} 52^{\circ} 32^{\prime} \mathrm{W}$, Uruguay): Canada (Newfoundland, Grand Bank, $43^{\circ} 30^{\prime} \mathrm{N}$ ), United States (Florida, Alabama), Antilles, Venezuela (Aruba), Guyana, Surinam $\left(7^{\circ} 44^{\prime} \mathrm{N} 54^{\circ} 40^{\prime} \mathrm{W}\right)$, French Guiana, Brazil (Santa Catarina), Uruguay $\left(35^{\circ} 34^{\prime} \mathrm{S} 52^{\circ} 32^{\prime} \mathrm{W}\right)$. Eastern Atlantic (northern limit: south of Portugal; southern limit: South Africa): Portugal, Morocco (Casablanca), Gulf of Guinea, Gabon, Congo-Brazzaville, South Africa. Indo-West Pacific (northern limit: Bay of Bengal; southern limit: $34^{\circ} 00^{\prime} \mathrm{S} 151^{\circ} 43^{\prime} \mathrm{E}$, Australia): South Africa (Cape Point, Natal), Mozambique, Madagascar, Tanzania (Zanzibar), Somalia, Arabian Sca (Malabar coast, Gulf of Manaar), Bay of Bengal, Andaman Is.), Indonesia, Japan, Australia (New South Wales).

Ecological notes. The species has been reported from 200 and $1850 \mathrm{~m}$, but occurs frequently at depths between 400 and $900 \mathrm{~m}$.

\section{Benthesicymus bartletti Smith, 1882}

\section{(Figures 1D,5)}

Benthesicymus Bartletti Smith, 1882.

Benthesicymus bartletti: Burkenroad, 1936: 47; Crosnier and Forest, 1969: 548; 1973: 275.

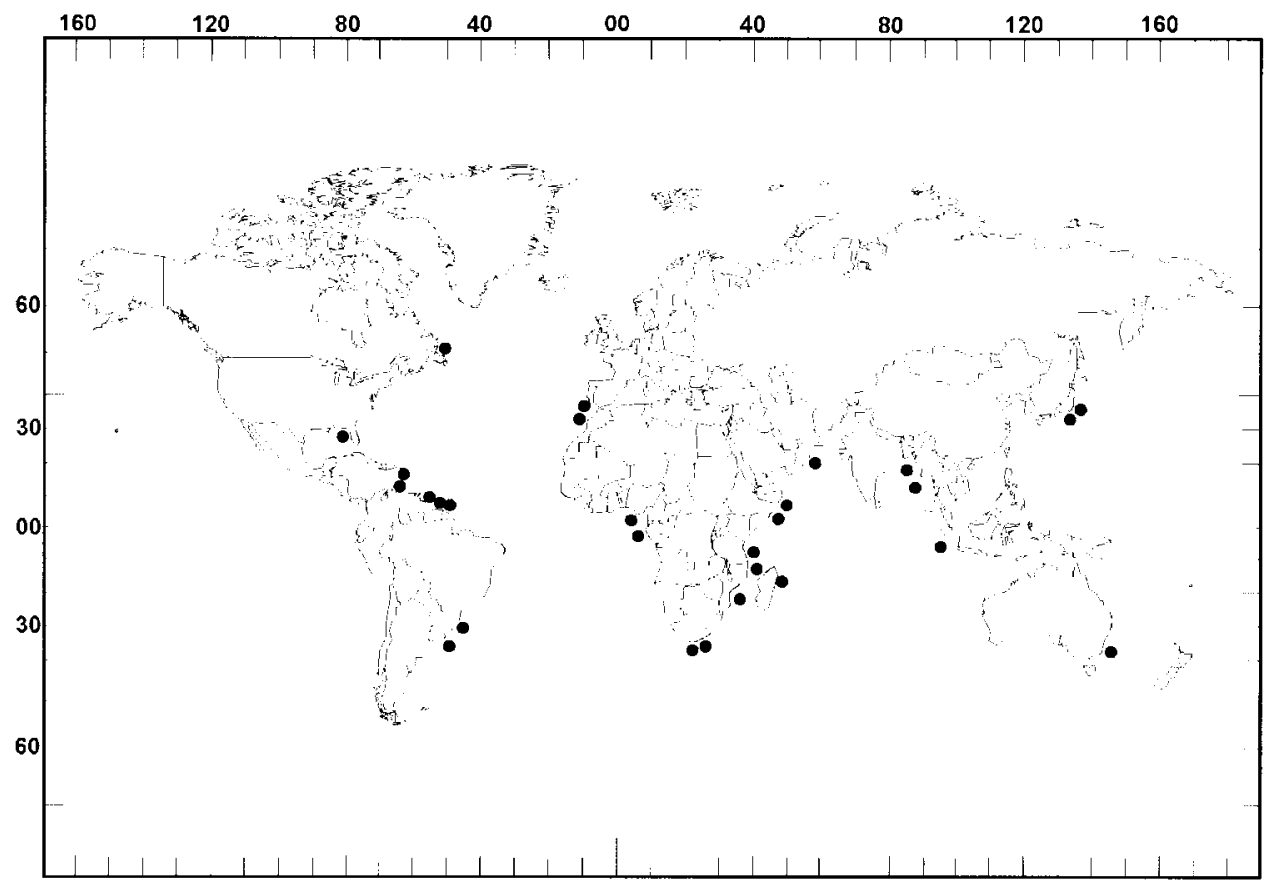

FIG. 4. Geographical distribution of Plesiopenaeus edwardsianus (Johnson, 1867). 
Material examined. Colombia: San Bernardo I., Oregon, st. 11240, $1.27 \mathrm{~m}, 2 \hat{\delta}$, $18.0 \mathrm{~mm}, 1$ ? , damaged specimen; (NHM 1977.535). Brazil: Rio de Janeiro, (22 $41^{\prime} \mathrm{S}$ $40^{\circ} 08^{\prime} \mathrm{W}$ ), W. Herwig, st. 103, $1200 \mathrm{~m}, 1 \overbrace{}^{\star}, 33.0 \mathrm{~mm}, 1$ †, $30.4 \mathrm{~mm}$, (INIDEP-690); $\left(22^{\circ} 41^{\prime} \mathrm{S} 40^{\circ} 08^{\prime} \mathrm{W}\right)$, W. Herwig, st. $103,1200 \mathrm{~m}, 1$ \% $, 33.0 \mathrm{~mm}, 2$ \% $31.4-34.2 \mathrm{~mm}$, (FURG-1604). Santa Catarina, (26 $47^{\prime} \mathrm{S} 45^{\circ} 57^{\prime} \mathrm{W}$ ), W. Herwig, st. $118,1200 \mathrm{~m}$, 1 \%, $29.6 \mathrm{~mm}$, (FURG-1605); (28 $34^{\prime} \mathrm{S} 46^{\circ} 33^{\prime} \mathrm{W}$ ), W. Herwig, st. 120, $1200 \mathrm{~m}, 1$ \%, $25.7 \mathrm{~mm}$, (INIDEP-1066); (28 $\left.34^{\prime} \mathrm{S} 46^{\circ} 33^{\prime} \mathrm{W}\right)$, W. Herwig, st. 120, $1200 \mathrm{~m}, 1 \delta^{\circ}$, $26.4 \mathrm{~mm}$, (FURG-1606).

Type-locality. East coast of United States, $39^{\circ} 45^{\prime} \mathrm{N} 70^{\circ} 55^{\prime} \mathrm{W}$.

Distribution. Western Atlantic (northern limit: Canada, $45^{\circ} 53^{\prime} \mathrm{N} 65^{\circ} 35^{\prime} \mathrm{W}$; southern limit: Santa Catarina, 28 $34^{\prime} \mathrm{S} 46^{\circ} 33^{\prime} \mathrm{W}$, Brazil): Canada, United States (Virginia), Bahama, Belize (16 $\left.58^{\prime} \mathrm{N} 87^{\circ} 53^{\prime} \mathrm{W}\right)$, West Caribbean (Rosalind Bank, $6^{\circ} 35^{\prime} \mathrm{N} 80^{\circ} 04^{\prime} \mathrm{W}$; Cayo Hobbies, $16^{\circ} 26^{\prime} \mathrm{N} 83^{\circ} 31^{\prime} \mathrm{W}$ ), Honduras (Punta Patuca, $16^{\circ} 26^{\prime} \mathrm{N} 84^{\circ} 24^{\prime} \mathrm{W}$ ), Panama (Gulf los Mosquitos, $9^{\circ} 05 \mathrm{~N} 81^{\circ} 18^{\prime} \mathrm{W}$; Mulatas Is., $9^{\circ} 00 \mathrm{~N} 77^{\circ} 25^{\prime} \mathrm{W}$ ), Colombia (Punta Caribana, Gulf of Darien, $8^{\circ} 56^{\prime} \mathrm{N} 76^{\circ} 53 \mathrm{~W}$, San Bernardo Is., $9^{\circ} 58^{\prime} \mathrm{N} 76^{\circ} 29^{\prime} \mathrm{W}$ ), Venezuela (La Orchilla I., $11^{\circ} 47^{\prime} \mathrm{N} 66^{\circ} 6^{\prime} \mathrm{W}$ ), Tobago (Man o'War Bay, $11^{\circ} 30^{\prime} \mathrm{N} 60^{\circ} 14^{\prime} \mathrm{W}$; Charlotteville, $11^{\circ} 37^{\prime} \mathrm{N} 60^{\circ} 59 \mathrm{~W}$ ), Guyana (Georgetown, $8^{\circ} 14 \mathrm{~N} 57^{\circ} 38^{\prime} \mathrm{W}$ ), Surinam, French Guiana (Fleuve Tracoubo, $7^{\circ} 31 \mathrm{~N}$ $53^{\circ} 11^{\prime} \mathrm{W}$ ), Brazil (Rio de Janeiro, $22^{\circ} 41^{\prime} \mathrm{S} 40^{\circ} 08^{\prime} \mathrm{W}$; Santa Catarina, $28^{\circ} 34^{\prime} \mathrm{S}$ $46^{\circ} 33 \mathrm{~W}$ ). Eastern Atlantic (northern limit: Açores I.; southern limit: Congo, $5^{\circ} 10^{\prime} \mathrm{S}$ ): Açores Is., Morocco, Canary Is., Cape Verde Is., Mauritania, Gabon, Congo. IndoWest Pacific (northern limit: North-Pacific, $37^{\circ} 49^{\prime} \mathrm{N} 166^{\circ} 47^{\prime} \mathrm{W}$; southern limit: Philippines): Bay of Bengal, Philippines, North-Pacific $\left(37^{\circ} 49^{\prime} \mathrm{N} 166^{\circ} 47^{\prime} \mathrm{W}\right)$.

Ecological notes. The occurrence of this shrimp on the coast of Rio de Janeiro and Santa Catarina are the first records for Brazil. The species occurs at depths

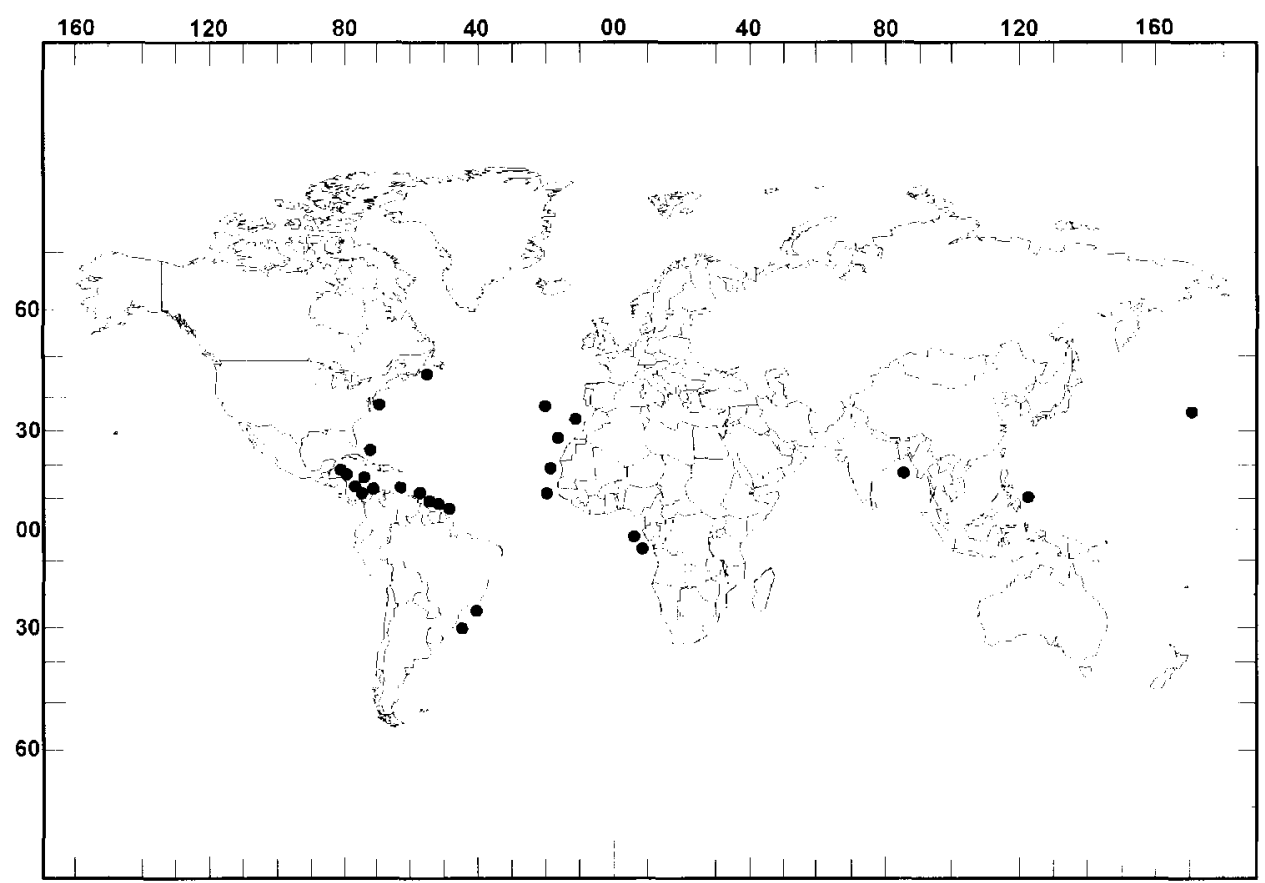

FIG. 5. Geographical distribution of Benthesicymus bartletti Smith, 1882. 
between 609 and $5777 \mathrm{~m}$, but its preferential range of depth is between 600 and $1800 \mathrm{~m}$.

\section{Bentheogennema intermedia (Bate, 1888)}

(Figures 1E,6)

Gennadas intermedius Bate, 1888: 343; Kemp, 1909: 723.

Bentheogennema intermedia: Burkenroad, 1936: 56; Crosnier and Forest, 1973: 278; Crosnier, 1978: 30; 1985: 858 .

Material examined. Brazil: Pernambuco. ( $\left.8^{\circ} 37^{\prime} \mathrm{S} 34^{\circ} 28^{\prime} \mathrm{W}\right)$, Challenger, st. 120 , $1229 \mathrm{~m}, 1$ \%, $14.9 \mathrm{~mm}$, (NHM 1888.22).

Type-locality. Off Sierra Leone, $1^{\circ} 47^{\prime} \mathrm{N} 24^{\circ} 26^{\prime} \mathrm{W}$, Africa.

Distribution. Western Atlantic (northern limit: Bermudas; southern limit: Pernambuco, $8^{\circ} 37^{\prime} \mathrm{S} 34^{\circ} 28^{\prime} \mathrm{W}$, Brazil ): Bermudas, United States (Florida), Bahamas, Gulf of Mexico, Brazil (Pernambuco). Eastern Atlantic (northern limit: $50^{\circ} \mathrm{N}$; southern limit: Cape Town, South Africa): Spain (Atlantic coast), Portugal, Açores Is., Madeira Is., Morocco, Canary Is., Sierra Leone, Gabon, Congo, Zaire, South Africa. Indo-West Pacific (northern limit: Japan; southern limit: East London, South Africa): South Africa (East London, Natal, Durban), Mozambique, Madagascar (Nosy Be), Tanzania (Zanzibar), Maldive Is., Arabian Sea, Japan, Hawaii. Eastern Pacific: Mexico (Baja California).

Ecological notes. The species occurs at depths between 900 and $4360 \mathrm{~m}$.

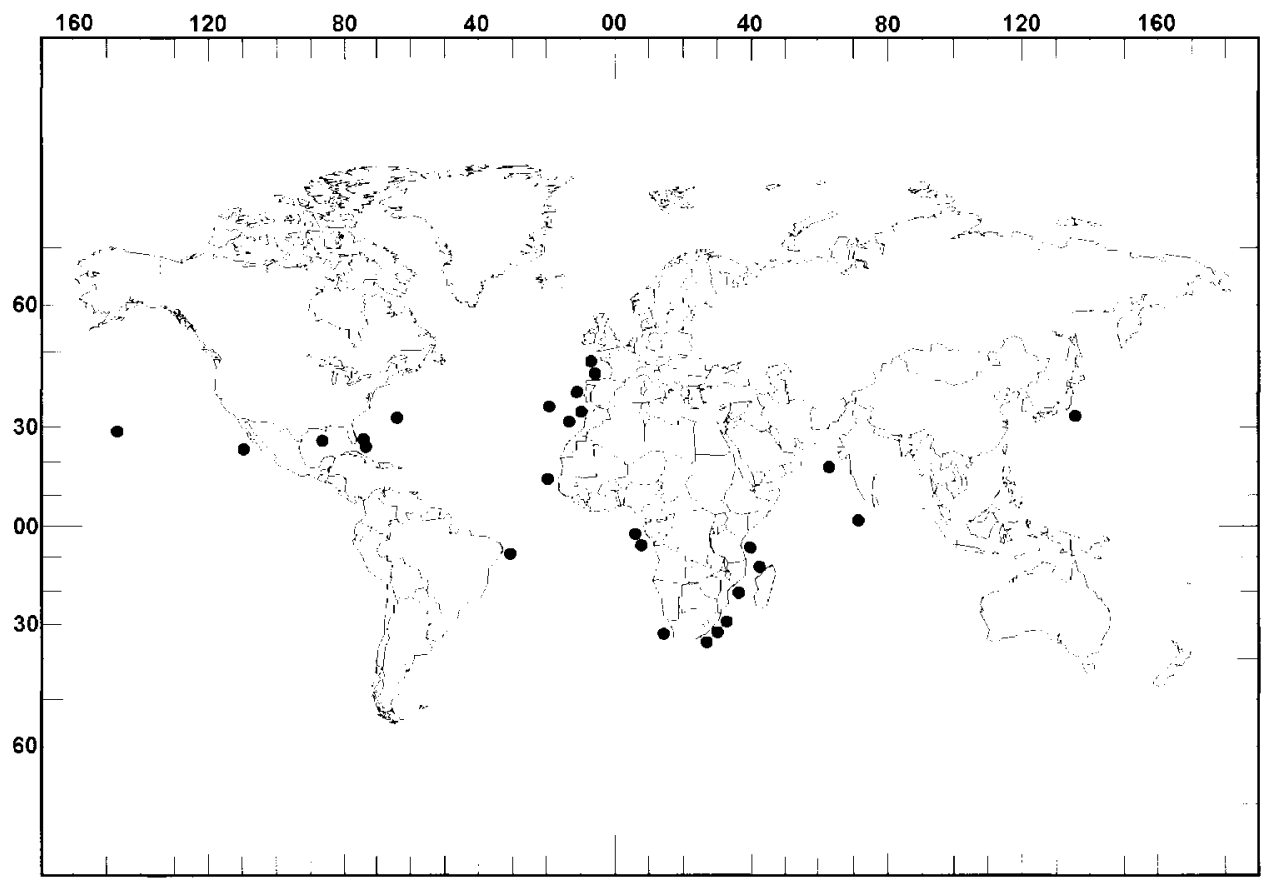

FIG. 6. Geographical distribution of Bentheogennema intermedia (Bate, 1888). 
Gennadas brevirostris Bouvier, 1905

(Figures $1 \mathrm{~F}, 7$ )

Gennadas brevirostris Bouvier, 1905: 748; Burkenroad, 1936: 65; Crosnier and Forest, 1969: 549; 1973: 283.

Material examined. Europe: Portugal ( $\left.40^{\circ} 49^{\prime} \mathrm{N} 11^{\circ} 18^{\prime} \mathrm{W}\right)$, W. Herwig, st. 3/III, $1 \%, 9.4 \mathrm{~mm}, 1+9.4 \mathrm{~mm}$, (FURG-1765). $\Lambda$ frica: Sahara $\left(20^{\circ} 38^{\prime} \mathrm{N} 22^{\circ} 32 \mathrm{~W}\right.$ ), Discovery, st. $7060,1000 \mathrm{~m}, 2$, $7.17 .4 \mathrm{~mm}$, (NHM 1971.98); (20 $38^{\prime} \mathrm{N} 22^{\circ} 32^{\prime} \mathrm{W}$ ), Discovery, st. $7060,1000 \mathrm{~m}, 1 \hat{\delta}, 7.7 \mathrm{~mm},(\mathrm{NHM} 1971.99)$; Mauritania $\left(20^{\circ} 06^{\prime} \mathrm{N}\right.$ $21^{\circ} 40 \mathrm{~W}$ ), W. Herwig, st. 10/III, 1 \%, $9.4 \mathrm{~mm}, 1$, $8.8 \mathrm{~mm}$, (FURG-1760); (16 $27^{\circ} \mathrm{N}$ $06^{\circ} 55^{\prime} \mathrm{W}$ ), W. Herwig, st. $1 / 1 \mathrm{II}, 2340 \mathrm{~m}, 1 \delta, 6.1 \mathrm{~mm}, 1 \%, 10.6 \mathrm{~mm}$, (INIDEP); $\left(16^{\circ} 11^{\circ} \mathrm{N} 22^{\circ} 24^{\prime} \mathrm{W}\right)$, W. Herwig, st. $11 / \mathrm{II}, 1955 \mathrm{~m}, 1 \stackrel{\circ}{\circ}, 8.5 \mathrm{~mm}, 1 \%, 5.0 \mathrm{~mm}$, (INIDEP-991); Guinea Bissau (12 27 N $\left.6^{\circ} 55^{\prime} \mathrm{W}\right)$, W. Herwig, st. 1/III, $2340 \mathrm{~m}, 1$ oे, $10.8 \mathrm{~mm}, 2$, $10.6-12.8 \mathrm{~mm}$, (FURG-1749); (1207’ $\left.23^{\circ} 07^{\prime} \mathrm{W}\right)$, W. Herwig, st. 12/2, 1944 m, 2 j, 7.4-8.9 mm, (FURG 1756). Brazil. Penedos de Säo Pedro e Säo Paulo, $\left(0^{\circ} 20^{\prime} \mathrm{N} 25^{\circ} 20^{\prime} \mathrm{W}\right)$, W. Herwig, st. $15 / \mathrm{I}, 1955 \mathrm{~m}, 1$ \% $7.3 \mathrm{~mm}$, (INIDEP); (6 $\left.6^{\circ} 06^{\prime} \mathrm{S} 27^{\circ} 00^{\prime} \mathrm{W}\right)$, W. Herwig, st. 18/III, $2042 \mathrm{~m}, 1$ j, $11.9 \mathrm{~mm}$, (FURG-1745). Alagoas, $\left(9^{\circ} 43^{\prime} \mathrm{S} 27^{\circ} 07^{\prime} \mathrm{W}\right)$, W. Herwig, st. $19 / 1,1930 \mathrm{~m}, 1$ ㅇ, $8.6 \mathrm{~mm}$,

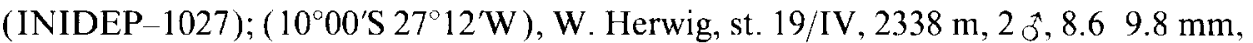

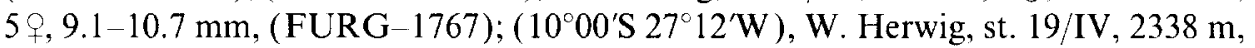
$1 \hat{0}, 12.1 \mathrm{~mm}$, (FURG-1748). Espírito Santo, $\left(21^{\circ} 04^{\prime} \mathrm{S} 30^{\circ} 12^{\prime} \mathrm{W}\right.$ ), W. Herwig, st.

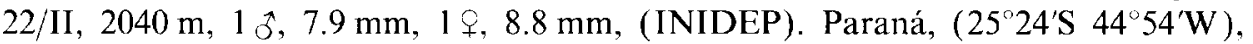
W. Herwig, st. $83,500 \mathrm{~m}, 1$ \% $8.3 \mathrm{~mm}$, (INIDEP). Santa Catarina, $\left(26^{\circ} 43^{\prime} \mathrm{S}\right.$ $45^{\circ} 57^{\prime} \mathrm{W}$ ), W. Herwig, st. $117,800 \mathrm{~m}, 3$, 10.2-12.5 mm, (FURG-1750); $\left(26^{\circ} 43^{\prime} \mathrm{S}\right.$ $\left.45^{\circ} 57^{\prime} \mathrm{W}\right)$, W. Herwig, st. $117,800 \mathrm{~m}, 1 \hat{j}, 10.7 \mathrm{~mm}, 29,9.4-9.9 \mathrm{~mm}$,

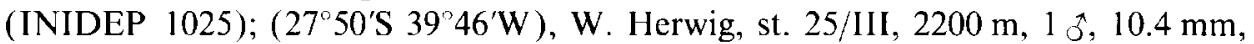
(INIDEP). Rio Grande do Sul, (29 $\left.58^{\prime} \mathrm{S} 42^{\circ} 27^{\prime} \mathrm{W}\right)$, W. Herwig, st. 26/II, $1945 \mathrm{~m}, 2$, 7.7-8.7 mm, (FURG-1751); (29 $\left.58^{\prime} \mathrm{S} 42^{\circ} 27^{\prime} \mathrm{W}\right)$, W. Herwig, st. 26/II, $1945 \mathrm{~m}$, 2 \%, 8.1-8.2 mm, ( INIDEP-1329); (30 $01^{\prime} \mathrm{S} 42^{\circ} 30^{\circ} \mathrm{W}$ ), W. Herwig, st. 26/III, $2042 \mathrm{~m}$, $2 \hat{\jmath}, 7.8-8.0 \mathrm{~mm},\left(\right.$ FURG-1746); ( $32^{\circ} 11^{\prime} \mathrm{S} 45^{\circ} 08^{\prime} \mathrm{W}$ ), W. Herwig, st. $27 / 1 \mathrm{II}, 2230 \mathrm{~m}$, 1 ?, $8.8 \mathrm{~mm}$, (FURG-1747). Uruguay: $\left(35^{\circ} 16^{\prime} \mathrm{S} 49^{\circ} 26^{\prime} \mathrm{W}\right)$, W. Herwig, st. 29/III, $2333 \mathrm{~m}, 1$ \%, $9.0 \mathrm{~mm}$, (INIDEP).

Type-locality. Saint Lucia, Lesser Antilles.

Distribution. Western Atlantic (northern limit: Cape Farewell, Greenland; southern limit: Uruguay, 35 $16^{\prime} \mathrm{S} 49^{\circ} 26^{\prime} \mathrm{W}$ ): Greenland (Cape Farewell), Lesser Antilles (Saint Lucia); Brazil (Penedos de São Pedro e São Paulo, Alagoas, Espírito Santo, Paraná, Santa Catarina, Rio Grande do Sul), Uruguay. Eastern Atlantic (northern limit: Bay of Biscay; southern limit: $31^{\circ} 21^{\prime} \mathrm{S} 9^{\circ} 45^{\prime} \mathrm{E}$ South Africa): France (Bay of Biscay), Spain (Bay of Biscay, Cadiz Bay), Morocco, Sierra Leone ( $8^{\circ} 58^{\prime} \mathrm{N}$, $16^{\circ} 27$ W), Gulf of Guinea, Congo, Gabon, Angola, South Africa (off Cape Town).

Ecological notes. The occurrence of this species along the coasts of Brazil has not been previously reported and it occurs at depths between 130 and $3500 \mathrm{~m}$.

\section{Conclusions}

The family Aristeidae is represented by six species in Brazil (Aristaeomorpha foliacea; Hepomadus tener; Plesiopenaeus edwardsianus; Benthesicymus bartletti; Gennadas brevirostris and Bentheogennema intermedia). The South America Atlantic coast species list is comprised of the Brazilian species and three species from Argentina (Plesiopenaeus armatus; Benthesicymus brasiliensis; Pseudaristeus 


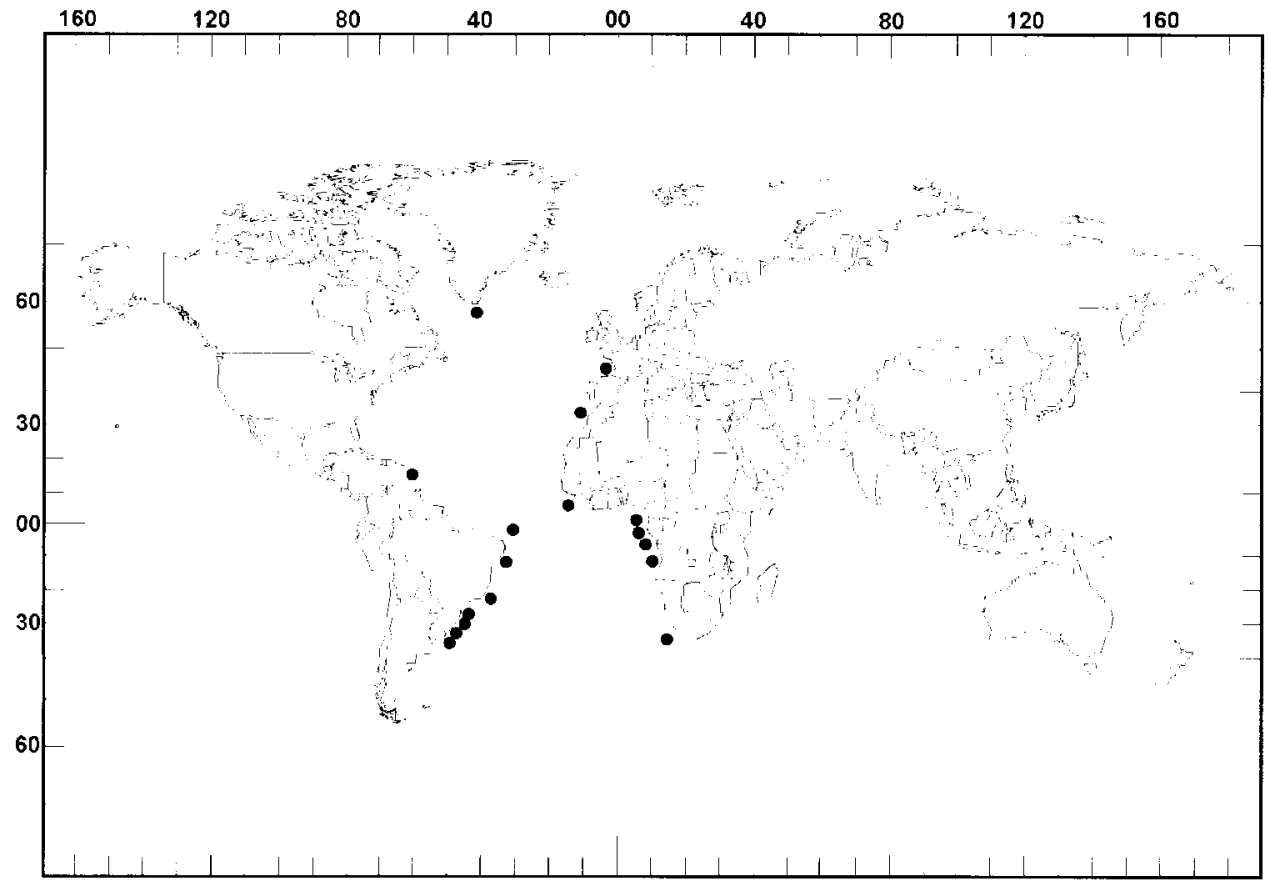

FIG. 7. Gcographical distribution of Gennadas brevirostris Bouvier, 1905.

speciosus). Further investigation in deep water will probably change and complete the list and the geographic distributions of the species.

The analysis of the Brazilian records showed that all species are widely distributed in the Atlantic and Indo-West Pacific oceans and the distribution pattern is similar among them. Hepomadus tener occurs in the Western Atlantic and Indo-West Pacific but Gennadas brevirostris is restricted to the Atlantic. Bentheogennema intermedia is the only species recorded in the Eastern Pacific. The latitudinal distribution ranges between $50^{\circ} \mathrm{N}$ and $40^{\circ} \mathrm{S}$. Only Gennadas brevirostris occurs out of this range, collectcd off Cape Farewell, Greenland.

\section{Acknowledgements}

The author would like to thank Dr Gustavo Augusto S. de Melo and Paul F. Clark (Natural History Museum) for the critical reading and the review of the English version of the manuscript.

\section{References}

Bate, C. S., 1881, On the Penacidac, Annals and Magazine of Natural History, London, series $5,8,169-196$.

BAtr, C. S, 1888, Report on the Crustacea Macrura collected by II. M. S. Challenger during the years 1873-76, Report of the Scientific Results of the Voyage of H. M. S. Challenger, Zoology, London, 24, 1942.

Bouvier, E. L, 1905, Sur les Macrourcs Nagcurs (abstraction faite des Carides), Recueillis par les Expéditions Américaines du Hassler et du Blake, Compte Rendu Hebdomadaire des Séances de l'Académie des Sciences, Paris, 141, 746-749.

Bullis, H. R., JR. and Thompson, J. R., 1959, Shrimp exploration by the M/V Oregon along the Northeast coast of South America, Commercial Fisheries Review, Washington, 21 (2), 1-9. 
Burkinrond, M. D., 1936, The Aristeidae, Solenoceridae and pelagic Penaeinae of the Bingham oceanographic collection, Bulletin of the Bingham Oceanographic Collection, New Haven, 5 (2), 1-151.

Coelho, P. A. and Ramos, M. A., 1972, A constituição e a distribuição da fauna de decápodos do litoral leste da America do Sul entre as latitudes de $5^{\circ} \mathrm{N}$ e $39^{\circ} \mathrm{S}$, Trabalhos do Instituto Oceanográfico da Universidade Federal de Pernambuco, Recife, 13, 133-236.

Crosnier, A., 1976, Données sur les Crustacés Décapodes captures par M. Paul Gueze à l'íle de la Réunion lors d'essais de pêche en eau profonde, Travaux et Documents de I'O.R.S.T.O.M., Paris, 47, 225-256.

Crosnier, A., 1978, Crustacés Décapodes Pénéides Aristeidae (Benthesicyminac, Aristeinae, Solenocerinae), Faune de Madagascar, Paris, 46, 1-197.

Crosnier, A., 1985, Crevettes pénéides d'eau profonde recoltées dans l'océan Indien lors des campagnes Benthedi, Safari I et II, MD 32/Réunion, Bulletin du Muséum d'Ilistoire Naturelle, Paris, Serie 4, Section A, 7 (4), 839-877.

Crosnier, A., 1989, Benthesicymidae, Aristeidae, Solenoceridae (Crustacea, Penaeoidea), Résultats des Campagnes MUSORSTOM, Paris, 5, 37-67.

Crosniter, A. and Fortst, J., 1969, Note preliminaire sur les peneides recuillis par L'Ombango', au large du plateau continental, du Gabon à l'Angola (Crustacea, Decapoda, Natantia), Bulletin du Muséum National d'Histoire Naturelle, 41 (2), 544 554.

Crosnier, A. and Forest, J., 1973, Les crevettes profondes de l'Atlantique Oriental Tropical, Faune Tropicale, 9, 1409.

ItUSARrY, E., 1984, Taxonomia y distribucion de los crustaceos (Decapoda) en el Frente Maritimo Uruguayo para los meses de febrero y marzo de 1982 (cruceros 820105 del B/P LEREZ), Tesis de Licenciatura en Oceanografia Biológica, Montevideo, Universidad de La Republica, $107 \mathrm{pp}$.

Johnson, J. Y., 1867, Descriptions of a new genus and a new species of macrurous decapod crustaceans belonging to the Penaeidae, discovered at Madeira, Proceedings of the Zoological Society of London, 1867, 875-901.

Kr.MP, S., 1909, The decapods of the genus Gennadas collected by H. M. S. 'Challenger', Proceedings of the Zoological Society of London, 1909, 718-730.

Pérez-Farfante, I., 1987, Revision of the gamba prawn genus $P_{\text {seudaristeus, with description }}$ of the new species (Crustacea: Decapoda: Penaeoidea), Fishery Bulletin of the National Oceanic and Atmospheric Administration, 85 (2), 311-338.

Ramos-Porto, M., Coelho, P. A. and Souzh, S. T., 1989, Sinopse dos crustáceos decápodes brasileiros (Familias Penaeidae, Solenoceridae, Sicyoniidae). Trabalhos do Instituto de Biologia Maritima e Oceanografia da Universidade Federal de Pernambuco, Recife, 20, 219234.

Risso, A., 1827, Histoire Naturelle des Principales Productions de l'Europe Méridionale et particulièrement de Celles des Environs de Nice et des Alpes-Maritimes, Vol. 5, 403 pp.

Smith, S. I., 1882, Report on the Crustacea. Part I. Decapoda. Reports on the results of dredging, under the supervision of Alexander Agassiz, on the east coast of the United States, during the summer of 1880 , by the U.S. Coast Survey steamer Blake, Bulletin of the Museum of Comparative Zoology, 10, 1-108.

Smith, S. I., 1884, Report on the decapod Crustacea of the Albatross dredging off the east coast of the United States in 1883, Report United States Commissioner of Fisheries, 10, $345-426$. 